\title{
Teaching prescribing skills to foundation trainees: a pilot study evaluating the impact of a prescribing training session for foundation year two doctors
}

\author{
Authors: Emily Willis, ${ }^{A}$ Steve Williams ${ }^{\mathrm{B}}$ and Paul Baker ${ }^{\mathrm{A}}$
}

\section{Aims}

To evaluate the impact of a 'good prescribing practice' teaching session on the attitudes and prescribing practice of FY2 trainees in the North Western Deanery.

\section{Methods}

A 1-hour, interactive teaching session was delivered to FY2 trainees at two North Western teaching hospitals. Pre- and postteaching online questionnaires were used to gather quantitative and qualitative data from the study group and a control group of trainees (post-teaching questionnaires completed at 3 months post-teaching session). Trainees were asked to rate their competence in relation to the British Pharmacological Society (BPS) principles of good prescribing practice. Trainees were asked to reflect on their prescribing practice, including any incidents related to prescribing that they had encountered. Thematic analysis was carried out on free-text responses.

\section{Results}

66 trainees responded to the pre-teaching questionnaire. $\geq 59 \%$ of trainees rated themselves almost fully competent or fully competent in relation to the BPS principles of Good Prescribing Practice. 24 post-teaching questionnaires were completed. In the post-teaching questionnaire, $\geq 50 \%$ of trainees rated themselves almost fully competent or fully competent in relation to the BPS principles. $100 \%$ of trainees who attended the teaching session and completed post-teaching questionnaires found the session enjoyable and useful. Analysis of trainees' reflections on their prescribing identified a number of themes including: prescribing errors; anxiety about prescribing for particular patient groups, eg paediatrics; external factors influencing prescribing, eg pressure from senior colleagues; time constraints; and cost.

\section{Conclusions}

Trainees' perceived competence in relation to the BPS principles of good prescribing practice was high prior to the teaching session. The response rate was insufficient to determine whether the teaching session had had any significant impact on trainees' competence in relation to the BPS principles. The interactive nature of the teaching session was rated highly by trainees. Trainees recognise the external factors that influence their prescribing decisions and value the opportunity to reflect on their practice with peers. Future prescribing training needs to address the influence of external factors such as time, workload and pressure from colleagues on the prescribing decisions made by juniors.

\section{Conflict of interest statement}

No conflicts of interest identified. 